Article

\title{
Genetic Diversity and Genetic Structure of the Wild Tsushima Leopard Cat from Genome-Wide Analysis
}

\author{
Hideyuki Ito ${ }^{1,2, *} \mathbb{C}$, Nobuyoshi Nakajima ${ }^{3}$, Manabu Onuma ${ }^{3}$ and Miho Murayama ${ }^{2,3}$ \\ 1 Kyoto City Zoo, Kyoto 606-8333, Japan \\ 2 Wildlife Research Center, Kyoto University, Kyoto 606-8203, Japan; mmurayama@wrc.kyoto-u.ac.jp \\ 3 Wildlife Genome Collaborative Research Group, National Institute for Environmental Studies, \\ Tsukuba 305-8506, Japan; naka-320@nies.go.jp (N.N.); monuma@nies.go.jp (M.O.) \\ * Correspondence: itohide7@gmail.com; Tel.: +81-75-771-0211; Fax: +81-75-752-1974
}

Received: 2 July 2020; Accepted: 5 August 2020; Published: 7 August 2020

Simple Summary: The Tsushima leopard cat, Prionailurus bengalensis euptilurus, is a small regional population of the Amur leopard cat and is only found on Tsushima Island in Japan. A breeding program will require adequate information on parentage, kinship, and inbreeding for this population. Hence, there is an urgent need to develop this information in order to conserve the population and its genetic diversity. We performed GRAS-Di analysis to investigate the genetic diversity and genetic structure of the Tsushima leopard cat. We identified between 133 and 158 single-nucleotide polymorphism (SNP) markers in three different genotyping methods. These SNP markers can be used in identification of individuals and parentage. In addition, structure analysis using these markers demonstrated the similar genetic composition of the samples from 48 Tsushima leopard cats, and indicated Tsushima leopard cats have no subpopulations. We have provided genetic markers that are useful for conservation of the Tsushima leopard cat, such as individual identification and parentage. Moreover, we have also clarified units for conservation of the Tsushima leopard cat population from structure analysis.

\begin{abstract}
The Tsushima leopard cat (Prionailurus bengalensis euptilurus) lives on Tsushima Island in Japan and is a regional population of the Amur leopard cat; it is threatened with extinction. Its genetic management is important because of the small population. We used genotyping by random amplicon sequencing-direct (GRAS-Di) to develop a draft genome and explore single-nucleotide polymorphism (SNP) markers. The SNPs were analyzed using three genotyping methods (mapping de novo, to the Tsushima leopard cat draft genome, and to the domestic cat genome). We examined the genetic diversity and genetic structure of the Tsushima leopard cat. The genome size was approximately $2.435 \mathrm{~Gb}$. The number of SNPs identified was 133-158. The power of these markers was sufficient for individual and parentage identifications. These SNPs can provide useful information about the life of the Tsushima leopard cat and the pairings and for the introduction of founders to conserve genetic diversity with ex situ conservation. We identified that there are no subpopulations of the Tsushima leopard cat. The identifying units will allow for a concentration of efforts for conservation. SNPs can be applied to the analysis of the leopard cat in other regions, making them useful for comparisons among populations and conservation in other small populations.
\end{abstract}

Keywords: Tsushima leopard cat; GRAS-Di; genetic diversity; genome-wide analysis

\section{Introduction}

The leopard cat (Prionailurus bengalensis) is widely distributed throughout South Asia, East Asia, and Southeast Asia. The Leopard cat is classified as Least Concern by The International Union for 
Conservation of Nature (IUCN) Red List of Threatened Species [1]. The leopard cat is generally classified into 12 subspecies [2]. A recent genetic study suggested that the leopard cat has only four subspecies [3]. The Amur leopard cat (Prionailurus bengalensis euptilurus), which is one of the subspecies, is distributed in Far East Asia. The Tsushima leopard cat is found only in Tsushima, Japan (Figure 1) and was classified as a regional population of the Amur leopard cat in recent genetic study [3]. Wild Tsushima leopard cat populations are declining due to habitat fragmentation, depletion of feeding grounds, and deaths due to impacts with vehicles. The population in the wild is estimated to be less than 100 animals [4]. Therefore, the Tsushima leopard cat has been awarded National Nature Monument status and has been classified as a critically endangered species on the Japanese Red List. In 1995, the Japanese Ministry of the Environment established a protection and breeding program for this wild cat.

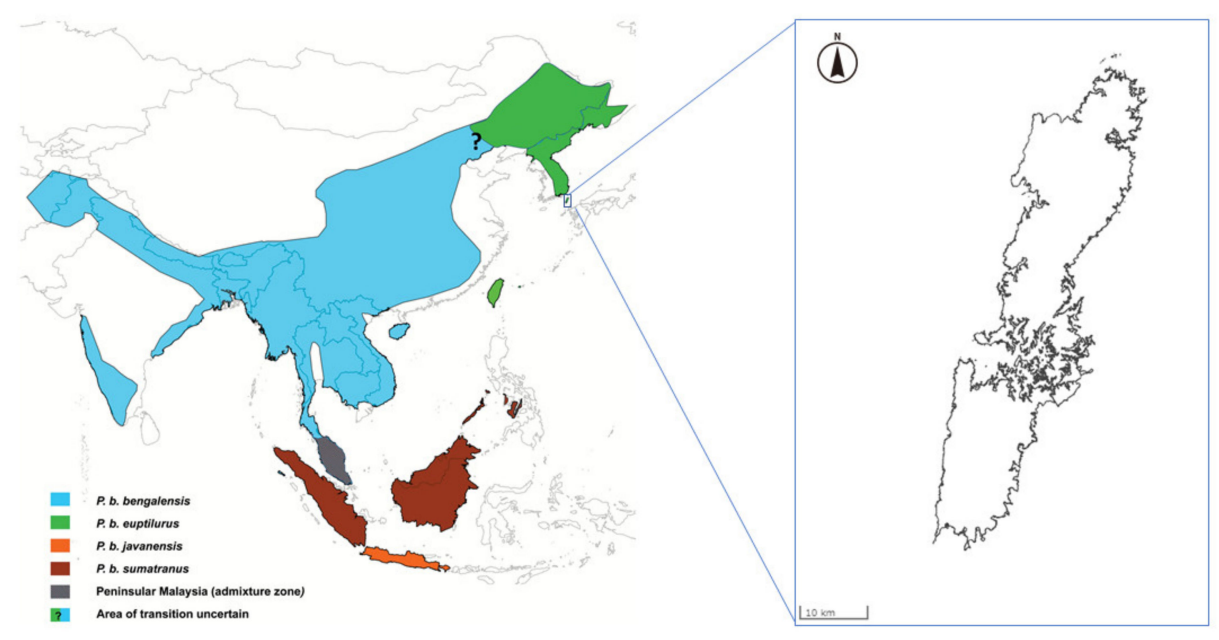

Figure 1. Distribution of the leopard cat subspecies (adapted from Patel et al. (2017) [3]) and location of Tsushima Island (modified map from the Geospatial Information Authority of Japan; https://maps.gsi.go.jp/).

The conservation of endangered species relies on detailed knowledge about nutrition, reproduction, husbandry, veterinary medicine, genetics, and more. Genetic information is especially important for small populations because they are susceptible to various genetic influences, including genetic drift, accumulation of deletion traits, and loss of genetic variations. The population of the Tsushima leopard cat is small, so a conservation strategy for this animal based on genetic information is important. Several genetic studies (e.g., mitochondrial DNA, Y chromosome DNA sequences, and microsatellite markers) have been performed, and the results have indicated that the Tsushima leopard cat has less genetic variation than other Asian leopard cat populations [5-7]. Generally, a captive breeding program can be established through genetic monitoring, usually requiring accurate parentage determination and estimation of individual kinship and inbreeding [8]. However, the identification of individuals in the Tsushima leopard population is difficult using microsatellite marker analysis due to the population's low genetic diversity [7], so more DNA markers must be developed to design an appropriate conservation program.

Microsatellite markers have many advantages, which have resulted in their wide use in the field of molecular ecology [9-11]. Single-nucleotide polymorphisms (SNPs) are enriched and relatively uniformly distributed throughout the genome, making them valuable genetic markers as well as microsatellites. Recently, advantages such as ease of genotyping, genetic stability, and suitability for automation have increased the use of SNPs [11-13]. Further, the advance of molecular genetics technology through next-generation sequencing (NGS) has made it possible to obtain extensive nucleotide sequences, enabling rapid and large-scale development of DNA markers. Moreover, more economical and efficient approaches using NGS have been developed, such as restriction site-associated 
DNA sequencing (RADSeq) [14], double digest RADSeq (ddRADSeq) [15], MIG-Seq (multiplexed ISSR genotyping by sequencing) [16], genotyping-in-thousands by sequencing (GT-Seq) [17,18], and genotyping by random amplicon sequencing, direct (GRAS-Di) $[19,20]$. These approaches enable simultaneous sequencing and genotyping of thousands of SNP markers, and they have been used in various species, including endangered species [18,21]. Although GRAS-Di has few applications thus far, GRAS-Di is useful in characterizing wildlife because it can function with the available low-quality DNA in a polymerase chain reaction (PCR)-based method and does not require a reference genome, similar to MIG-seq $[19,20]$. GRAS-Di is a technology that produces a sequence library by two PCRs using random primers and adapter sequences, and detects SNPs using NGS. GRAS-Di is expected to develop more SNPs than MIG-seq because it is a non-targeting method using short random primers, which is an advantage of this approach. The purpose of this study was to identify SNPs using genome-wide analysis with the GRAS-Di approach that would clarify the genetic diversity and structure of the wild Tsushima leopard cat.

\section{Materials and Methods}

\subsection{Sample Collection}

This study was conducted in strict accordance with the guidelines for ethics in animal research established by the Wildlife Research Center of Kyoto University. The Kyoto City Zoo approved the research (\#20170426). Muscle samples were obtained from 2004 to 2015 from 48 Tsushima leopard cats that had been either killed in road accidents or rescued from road accidents and later died. All muscle samples were stored in ethanol until DNA extraction using the QIAGEN DNeasy Blood and Tissue Kit (Qiagen, Hilden, Germany). DNA concentrations were measured using a NanoDrop spectrophotometer (Thermo Fisher Scientific, Waltham, MA, USA).

\subsection{Construction of the Draft Genome}

The isolated genomic DNA was used to construct short-insert libraries using TruSeq DNA PCR Free (350) (Illumina, San Diego, CA, USA), following standard protocols provided by Illumina. This was followed by 150-bp paired-end sequencing using a whole-genome shotgun sequencing strategy on the Illumina Hiseq X Ten platform. Library construction and sequencing took place at Macrogen Japan (Kyoto, Japan). Development of the draft genome used Genomic Workbench v. 11.01 (Qiagen). "Trim reads" were performed on all the read data. Quality trimming was set at $p=0.05$ and the read-through adapters were removed. Reads were mapped to the cat genome using "Map reads to reference" in the global alignment mode. Default values were used for the similarity fraction (0.8) and length fraction (0.5) as parameters. Next, those portions of coverage connecting 10 or more reads by mapping were defined as contigs. Furthermore, the "De novo assemble tool" was applied using the trimmed read data in the "Create simple contig sequence" mode. Word size was 60 . The process merged scaffolds and contigs using the "Join contig tool".

\subsection{GRAS-Di Analysis}

GRAS-Di was developed by the Toyota Motor Corporation (Aichi, Japan) (patent ID P2018042548A) [20,22]. Library preparation and sequencing were done by Bioengineering Lab. Co., Ltd., under a license agreement. The library was constructed using two-step tailed polymerase chain reaction (PCR). The first group of PCR primers included Illumina Nextera adaptor sequences plus three-base random oligomers, and the second group of PCR primers included the Illumina multiplexing dual index and P7/P5 adapter sequence. The first PCR was performed in a $25 \mu \mathrm{L}$ reaction volume containing $5.0 \mu \mathrm{L}$ of $5 \times$ PrimeStar buffer ( $\mathrm{Mg}^{2+}$ plus) (Takara) (Shiga, Japan), $2.0 \mu \mathrm{L}$ of dNTP mixture (2.5 mM of each), $10 \mu \mathrm{L}$ of random primer mix $(100 \mu \mathrm{M}), 0.25 \mu \mathrm{L}$ of PrimeStar HS DNA polymerase $(2.5 \mathrm{U} / \mu \mathrm{L})$ (Takara), $1 \mu \mathrm{L}$ of genome DNA $(15 \mathrm{ng} / \mu \mathrm{L})$, and $6.75 \mu \mathrm{L}$ of sterile water. The reaction conditions were initial denaturation at $98^{\circ} \mathrm{C}$ for $2 \mathrm{~min}$ and 30 cycles of $98^{\circ} \mathrm{C}$ for $10 \mathrm{~s}, 50^{\circ} \mathrm{C}$ for 
$15 \mathrm{~s}$, and $72{ }^{\circ} \mathrm{C}$ for $20 \mathrm{~s}$. The second PCR was performed in a $50 \mu \mathrm{L}$ reaction volume containing $10 \mu \mathrm{L}$ of $5 \times$ PrimeStar buffer ( $\mathrm{Mg}^{2+}$ plus), $4.0 \mu \mathrm{L}$ of dNTP mixture (2.5 mM of each), $1.25 \mu \mathrm{L}$ of P7 primer with 8 base index $(10 \mu \mathrm{M}), 1.25 \mu \mathrm{L}$ of P5 primer with 8 base index $(10 \mu \mathrm{M}), 0.5 \mu \mathrm{L}$ of PrimeStar HS DNA polymerase $(2.5 \mathrm{U} / \mu \mathrm{L}), 1.5 \mu \mathrm{L}$ of PCR product from the first PCR, and $31.5 \mu \mathrm{L}$ of sterile water. The reaction conditions were initial denaturation at $95^{\circ} \mathrm{C}$ for $2 \mathrm{~min}, 25$ cycles of $98{ }^{\circ} \mathrm{C}$ for $15 \mathrm{~s}, 55^{\circ} \mathrm{C}$ for $15 \mathrm{~s}$, and $72{ }^{\circ} \mathrm{C}$ for $20 \mathrm{~s}$, followed by a final extension period at $72{ }^{\circ} \mathrm{C}$ for $1 \mathrm{~min}$. The final PCR products were pooled, purified using the MiniElute PCR Purification Kit (Qiagen) following the manufacturer's protocol, and applied for sequencing on NextSeq 500 (Illumina) using the NextSeq 500/550 Mid Output Kit v. 2.5 (paired-end, 150 cycles) (Illumina).

Low-quality reads were trimmed using Sickle v. 1.33 [23]. The Nextera adaptor and other Illumina primer sequences were clipped with default settings. Trimmed reads shorter than $50 \mathrm{bp}$ were removed. Moreover, sequences after 50 bases were deleted using fastx_trimmer in FASTX-Toolkit [24].

\subsection{Genotyping}

SNP analyses were performed with three methods using Stacks v. 2.5 [25]. Stacks is a method developed for the RAD analysis and has also been applied to PCR-based methods such as MIG-Seq and GRAS-Di $[16,26]$. In the first method, the denovo_map pl mapping protocol (hereafter referred to as de_novo) was used. The denovo_map.pl included six core components: building loci (ustacks), creating a catalog of loci (cstacks), and matching samples back against the catalog (sstacks), transposing the data (tsv2bam), adding paired-end reads to the analysis and calling genotypes (gstacks), and population genomics analysis (populations). In the second and third methods, the domestic cat (Felis catus) genome (hereafter referred to as ref_cat) and the Tsushima leopard cat (hereafter referred to as ref_TLC) draft genome were each mapped as a reference genome using the ref_map pl protocol, which consisted of two components (gstacks and populations). With the first method (de_novo), SNP calling for each individual was done using the program denovo_map pl of Stacks v. 2.5 with the "-m 5" option (other options set to default) to delete and discard SNPs with read depth $<5$. The populations program of Stacks v. 2.5 was used at default settings. With the other two methods (ref_cat and ref_TLC), trimmed reads were mapped onto the reference genome of the domestic cat (Felis_catus_9.0) and the draft genome of the Tsushima leopard cat, respectively, using BWA-mem [27]. The SAM files thus obtained were converted to BAM files and sorted using SAMtools v. $1.4[28,29]$. Any region with coverage $<5$ was deleted by VariantBam v. 1.4.4 [30]. SNP calling for each individual was done using the program ref_map pl of Stacks v. 2.5. The populations program of Stacks v. 2.5 was used at default settings.

\subsection{Analysis of Genetic Diversity and Genetic Structure}

PLINK v. 2.0 [31] was used to filter the dataset so that it would contain SNPs found in at least $80 \%$ of individuals (-geno 0.2) and would exclude those from individuals with more than $20 \%$ missing genotypes (-mind 0.2). As an additional quality control step, low-confidence SNPs were filtered out using PLINK v. 2.0 in terms of the Hardy-Weinberg equilibrium (HWE) at $p<0.05$ and linkage disequilibrium between all possible pairs of loci. The output files from PLINK were converted to the Genepop format using PGDSpider v. 2.1.1.5 [32]. The expected heterozygosity $(\mathrm{He})$ and observed heterozygosity $(\mathrm{Ho})$, probability of identity (PID), PID among siblings (PID-sib), and probability of exclusion (PE) (when the other parent is known) were calculated using GenALEx v. 6.5 [33].

The SNP data were also analyzed using the program Structure in Strauto [34] using the admixture model to estimate population genetic structure. We conducted an analysis with 10 iterations for each population size $(K)$ of 1 to 10, and with Markov-chain Monte Carlo (MCMC) sampling running for 500,000 generations with an initial burn-in of 300,000 generations. The $K$ values described by Pritchard, et al. [35] and Evanno, et al. [36] $(\operatorname{Ln} P(K)$ and $\Delta K$, respectively) were then calculated to identify the most reasonable $K$ using the program Structure Harvester [37] in Strauto. The $\operatorname{Ln} P(K)$ is an estimate of the posterior probability of the simulation, and it is a log probability of the data at each $K . \Delta K$ is the rate of change in the log probability of data between successive $K$ values. 
Moreover, the most reasonable $K$ values were identified using a parsimony index described by Wang (2019) [38]. Runs were averaged and visualized using CLUMPAK [39]. The population structure was also examined by carrying out principal coordinate analysis (PCoA) using GenALEx v. 6.5 [33] to visualize the genetic relationship among different individuals in two dimensions.

\section{Results}

\subsection{Developing the Draft Genome}

For development of the draft genome of the Tsushima leopard cat, 905,091,004 reads were produced, and total read bases comprised $136.7 \mathrm{G}$ bp. The entire process was performed using Genomic Workbench v. 11.01. The developed assembly had a genome size of $2.435 \mathrm{~Gb}$ covered in 65,356 scaffolds with an average size and scaffold N50 of 37,262 bp and 152,598 bp, respectively (DDBJ Accession Number: BIMV01000001-BIMV01065356).

\subsection{Genetic Diversity}

In total, using GRAS-Di analysis, 71,634,742 raw reads with an average of 1,492,578 reads per sample were obtained for 48 individuals (DDBJ Accession Number: BIMV01000001-BIMV01065356). The three SNP data sets created using different methods resulted in specific numbers of individuals and loci (with the process for each of the three methods detailed in Table S1). After the filtering steps, the numbers of loci and individuals remaining for ref_TLC, ref_cat, and de_novo were 158 loci/42 individuals, 143 loci/42 individuals, and 133 loci/41 individuals, respectively.

Genetic diversity indices of the Tsushima leopard cat in three methods are summarized in Tables 1 and 2. Average values for $H o$ and $H e$ in all loci for ref_TLC, ref_cat, and de_novo were $0.084 / 0.088(0.000-0.429 / 0.024-0.037), 0.076 / 0.083(0.000-0.405 / 0.024-0.410)$, and 0.092/0.095 (0.000-0.405/0.024-0.0354), respectively. The cumulative PID values for all loci for ref_TLC, ref_cat, and de_novo were $1.7 \times 10^{-11}, 1.3 \times 10^{-9}$, and $1.9 \times 10^{-10}$, respectively. The cumulative PID-sib values for all loci for ref_TLC, ref_cat, and de_novo were $3.1 \times 10^{-5}, 2.6 \times 10^{-4}$, and $1.0 \times 10^{-4}$, respectively. The cumulative PE values (when the other parent is known) for all loci for ref_TLC, ref_cat, and de_novo were $0.9987,0.9965$, and 0.9977 , respectively.

Table 1. Genetic diversity indices of the Tsushima leopard cat in three genotyping methods.

\begin{tabular}{|c|c|c|c|c|c|}
\hline & & $N$ & $N e$ & Ho & He \\
\hline \multirow[t]{2}{*}{ ref_TLC } & Mean & 38.823 & 1.104 & 0.084 & 0.088 \\
\hline & $\mathrm{SE}$ & 0.153 & 0.008 & 0.007 & 0.006 \\
\hline \multirow[t]{2}{*}{ ref_cat } & Mean & 39.000 & 1.099 & 0.076 & 0.083 \\
\hline & SE & 0.150 & 0.009 & 0.007 & 0.006 \\
\hline \multirow[t]{2}{*}{ de_novo } & Mean & 38.098 & 1.114 & 0.092 & 0.095 \\
\hline & SE & 0.161 & 0.009 & 0.008 & 0.006 \\
\hline
\end{tabular}

$N$ : number of individuals, $\mathrm{Ne}$ : the number of effective alleles, $\mathrm{Ho}$ : observed heterozygosity, He: expected heterozygosity.

Table 2. Genetic diversity indices of the Tsushima leopard cat in three genotyping methods.

\begin{tabular}{cccc}
\hline & PID & PID-sib & PE \\
\hline ref_TLC & $1.7 \times 10^{-11}$ & $3.1 \times 10^{-5}$ & 0.9987 \\
ref_cat & $1.3 \times 10^{-9}$ & $2.6 \times 10^{-4}$ & 0.9977 \\
de_novo & $1.9 \times 10^{-10}$ & $1.0 \times 10^{-4}$ & 0.9964 \\
\hline
\end{tabular}

PID: cumulative probability of identity, PID-sib: cumulative PID among siblings, PE: cumulative probability of exclusion. 


\subsection{Genetic Structure within the Tsushima Leopard Cat Samples}

The most reasonable $K$ values from the three methods are displayed in Table 3 . The best $K$ value was $K=2$ in all three genotyping methods for Mean_LnP(K). Similarly, for parsimony, the best $K$ value in all methods was $K=1$. On the other hand, the $\Delta K, K=3$ in ref_TLC and de_novo and $K=2$ in ref_cat, showed different values for different methods. Structure analysis and principal coordinate analysis in the three methods are demonstrated in Figures 2 and 3, respectively. The bar plots of each individual for $K=2$ and $K=3$ with the greatest support from structure analysis are shown in Figure 2 . In the PCoA, most of the individuals in all three methods were plotted in proximity to each other, with only a few individuals further away (Figure 3). The percentages of variation explained by the first two axes in ref_TLC, ref_cat, and de_novo were $7.81 \% / 5.68 \%, 7.71 \% / 5.78 \%$, and 6.27\%/5.72\%, respectively.

Table 3. The best $K$ values in combinations three genotyping methods and three $K$ estimators.

\begin{tabular}{cccc}
\hline & ref_TLC & ref_cat & de_novo \\
\hline$\Delta K$ & 3 & 2 & 3 \\
Mean $\operatorname{Ln} P(K)$ & 2 & 2 & 2 \\
Parsimony & 1 & 1 & 1 \\
\hline
\end{tabular}

a)

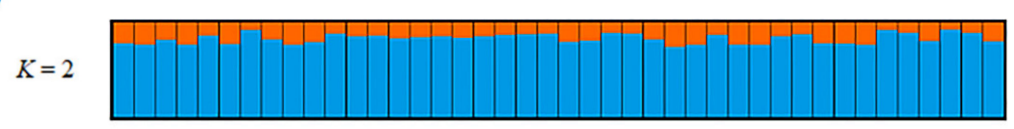

$K=3$

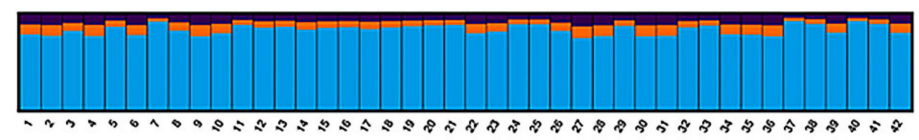

b)

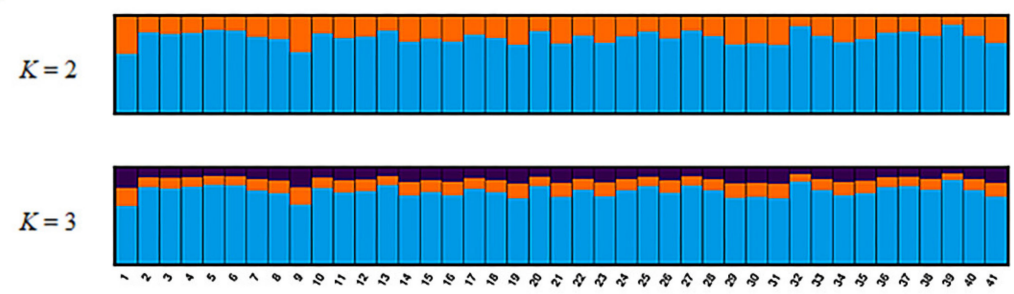

c)

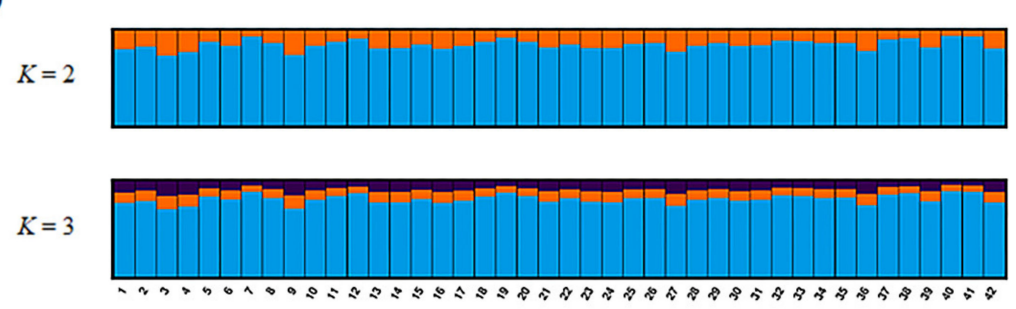

Figure 2. Bayesian analysis of the genetic structure in wild populations of the Tsushima leopard cat showing no deviation based on single-nucleotide polymorphism (SNP) markers. This figure was constructed by using CLUMPAK to align the 10 replicates for $K=2$ and $K=3$ for three genotyping methods (with all runs using the Markov-chain Monte Carlo method for 500,000 generations and an initial burn-in of 300,000 generations): (a) ref_TLC, (b) ref_cat, and (c) de_novo. 
a) ref_TLC

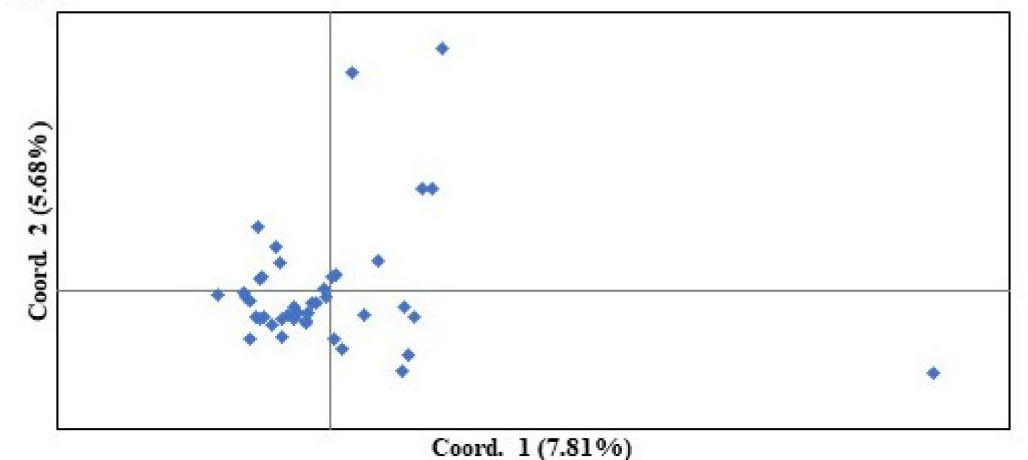

b) ref_cat

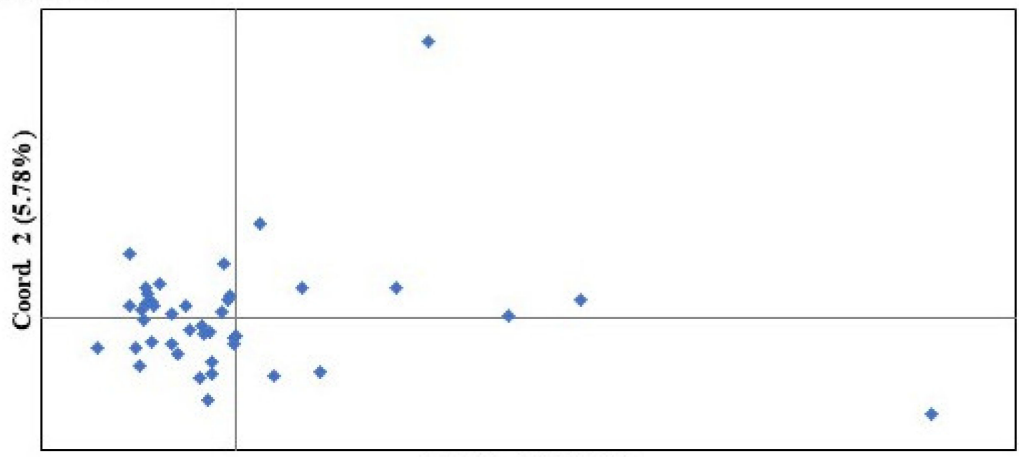

Coord. $1(7.71 \%)$

c) de_novo

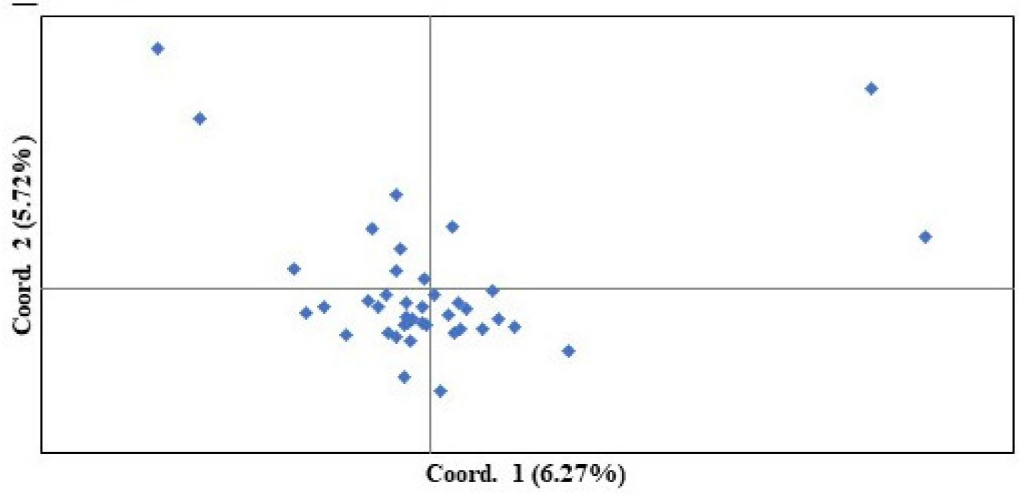

Figure 3. First and second components of principal coordinate analysis of SNP markers in the Tsushima leopard cat for three methods: (a) ref_TLC, (b) ref_cat, and (c) de_novo. Parentheses denote the percentages of variation explained.

\section{Discussion}

The Tsushima leopard cat is a regional population of the leopard cat in Japan, and it is a critically endangered species; several studies for conservation have been implemented [7,40-42]. The management of the population of the Tsushima leopard cat needs DNA markers that can not only determine individual genetic diversity but also identify individuals and clarify genetic relationships. In this study, we identified candidate SNP markers by GRAS-Di, and we compared analysis of GRAS-Di data using a de novo approach to analysis using a reference genome. 
The numbers of SNP candidates detected in output data from GRAS-Di were about the same for the methods ref_TLC $(n=4859)$ and ref_cat $(n=4868)$ and less for de_novo $(n=4330)$. After qualitative filtering, ref_TLC $(n=158)$ had the highest number, followed by ref_cat $(n=143)$ and de_novo $(n=133)$. The use of a closely related species as a reference was more useful for the detection of SNP candidates, even though the reference was not of the same species. The denovo_map.pl analysis is expected to identify more SNP markers than the ref_map.pl analysis, which considers only the reads mapped to the reference genome, as all reads are considered for marker identification $[43,44]$. On the other hand, it was reported that there are more SNPs that are discovered by using the reference genome [45]. In addition, the reference genome has the merit of separating false positive loci from the true loci and identifying microbial DNA contaminants, and it functions as a filter [46]. Our study showed the later result. These results suggest that coverage was lower, the Tsushima leopard cat draft genome was qualitatively sufficient as a reference, there is a close relationship between domestic cats and the Tsushima leopard cat, and there was a low level of contamination in the sample DNA. The numbers of markers detected after filtering in this study were low (ranging from 133 to 158) compared with another GRAS-Di study (ranging from 4457 to 8931) [19]. Low numbers of SNPs were detected because of (i) the platform used for the analysis, (ii) the analysis pipeline, (iii) the number of individuals studied (48 samples in this study versus 59-100 samples in the previous study) [19], (iv) the number of sequenced reads per individual, (v) the genome size of the species, and (vi) the lack of genetic diversity in the Tsushima leopard cat. The various options for filtering the programs Stacks and PLINK, used for bioinformatics analysis, have been shown to significantly affect the resulting number of SNPs [47,48]. However, the values of each option are not identical across all datasets [47], making it difficult to compare the assessment of genetic diversity with the number of SNPs detected. Therefore, the low SNP numbers found in the Tsushima cat population compared to other species do not indicate low genetic diversity.

In a previous study using 12 microsatellite markers, the genetic diversity indexes in the Tsushima leopard cat were extremely low (PID and PID-sib were $2.1 \times 10^{-1}$ and $4.5 \times 10^{-1}$, respectively), so this microsatellite marker set could not perform identification of individuals [7]. In contrast, in this study, values of PID and PID-sib in the three genotyping methods were low enough that individual identification was thought to be possible. In addition, the high cumulative $P E$ for the three methods indicated the possibility of identifying parentage in the Tsushima leopard cat. Therefore, the SNP marker set developed in this study can be applied to elucidate the animal life of the Tsushima leopard cat, including identification of individuals and estimation of kinship, and can be useful in conservation. In addition, in microsatellite analysis, it is necessary to have and share reference samples to compare microsatellite results among different laboratories. However, because SNP genotyping is easy to standardize among different laboratories, the SNPs discovered in this study can be useful for comparison of different research approaches (samples, machine, laboratory, and researcher).

Despite its high cost, for the Tsushima leopard cat, the best approach was to develop and use the draft genome. However, in non-model species, there is often no reference genome. Although both the de_novo and ref_cat methods were inferior to the ref_TLC version, they were considered useful for individual identification, parentage, and evaluation of genetic diversity. In the present study, the number of SNP markers was higher with the ref_cat method, which used the genome of the domestic cat, a closely related model species, as a reference, than in the de_novo method, but the de_novo method had higher genetic diversity indices. Mitochondrial DNA analysis estimated the divergence between F. catus and P. bengalensis to be 7.33 mya [49]. Considering these values and generation lengths for different species will lead to selecting better methods (de novo mapping or mapping to closely related species).

There were many SNPs that were excluded by deviations from the HWE (76.6\% in ref_TLC, $78.5 \%$ in ref_cat, and $64.0 \%$ in de_novo were removed) (Table S2). Testing for the HWE in populations serves as a quality control step in genetic analysis and is a very common tool for detecting errors in sequencing and genotyping [50-52]. Similarly, in studies with a large number of markers, such as the Genome-Wide 
Association Study (GWAS), the HWE has been treated as a baseline for quality control [53,54]. However, various factors such as mutation, natural selection, nonrandom crossing, genetic drift, and gene flow can cause deviations from the HWE [55]. For example, deviations may occur in populations that have experienced inbreeding or have experienced recent bottlenecks. In populations with inbreeding experience, the value of $\mathrm{Ho}$ is smaller than the value of $\mathrm{He}$, and in populations immediately after they undergo bottlenecking, the value of $\mathrm{He}$ is smaller than that of $H o$ (heterozygosity excess). Therefore, filtering by deviations from the HWE may blur the differences between significant deviations, such as those derived from evolutionary selection, and deviations due to genotyping errors. In this study, there was a large difference between mean $\mathrm{He}(0.313)$ and mean $H o(0.037)$ before HWE filtering. In addition to genotyping errors such as allele dropout, there may be many SNPs that misidentify a heterozygote as a homozygote due to low coverage. To derive a more accurate population structure, it is necessary to consider the effects of $H W E$ filtering methods and to decrease typing errors by increasing coverage.

The best $K$ values in the structure analysis were 1 to 3 , according to the genotyping method and the $K$ calculation method. In the analysis of population structure, $\Delta K, \operatorname{Mean} \_\operatorname{Ln} P(K)$, or both are often used to calculate best $K$ values. However, accurate estimation of $K$ is difficult, and using $\Delta K$ and Mean_LnP(K) may overestimate or underestimate the value of $K$ due to unbalanced sampling, low population differentiation, low marker information, hierarchical structure, and inbreeding $[38,56]$. Therefore, it is preferable to show plots of Mean_LnP $(K)$ and $\Delta K$, or to indicate structural bar plots of multiple $K$ values [56]. It is also recommended that multiple methods of results be compared (e.g., structure analysis with BAPS or PCA) [56]. In this study, the results of the bar plots at $K=2$ and $K=3$ suggest that the individuals have a similar genetic composition and that all individuals belong to the same population, which in turn means that the Tsushima leopard cat has no subpopulations. This study also used the parsimony index [38], which differs from $\Delta K$ and Mean_LnP(K), in calculating $K$. The parsimony method showed $K=1$ for all three genotyping methods, and this value was the most suitable in the dataset of this study. It has been reported that the parsimony index can calculate $K$ values that are more suitable than $\Delta K$ and $M e a n_{-} \operatorname{Ln} P(K)$ under various conditions [38], and since these can be calculated from the output file from Structure as well as $\Delta K$ and Mean $\_n P(K)$, investigating $K$ values together with $\Delta K$ and Mean $\_n P(K)$ was considered effective.

In the PCoA results, data for most of the individuals were plotted in similar locations and no division within the population could be confirmed. However, the data for a few individuals (1-4) were located slightly apart from the others in the plot. Although the genetic differences were small, these individuals were thought to retain a slightly different genetic composition. It is thus possible to view these as high-priority individuals for captive breeding. In addition, increasing the sample size would clarify whether subpopulations exist or not. Breeding of the Tsushima leopard cat has been carried out in captive conservation with various genetic studies. However, because the genetic relationship between founders in captive populations is unknown, breeding plans are designed assuming that there is no kinship between the founders. Therefore, it is important to note the difference between genetic diversity based on pedigree and genetic diversity based on genetic analysis [57]. This is the case especially when the wild population is small, such that the difference between the two genetic diversities may be large [57]. The Tsushima leopard cat also has a small population size, so the genetic diversity it actually retains is likely to be lower than the genetic diversity based on pedigrees, and a more careful breeding plan is needed. In the future, the analysis of the captive population using these SNP markers can identify high-priority breeding founders and strains.

\section{Conclusions}

We identified more than one hundred SNP markers in the endangered Tsushima leopard cat by GRAS-Di analysis, which is one of the genome-wide analyses. These markers can be used in individual identification and parentage. We indicated that the Tsushima leopard cat population has no sub-population using these markers, this result can lead to the optimization of conservation efforts. 
Gras-Di analysis was useful for SNP discovery in endangered species. Our results will provide useful information for the conservation of the Tsushima leopard cat.

Supplementary Materials: The following are available online at http:/www.mdpi.com/2076-2615/10/8/1375/s1, Table S1: The numbers of loci and individuals at each filtering steps Table S2: Mean $\operatorname{LnP}(\mathrm{K})$, delta $\mathrm{K}$, and parsimony index at each $\mathrm{K}$ in three genotyping methods.

Author Contributions: Conceived and designed the experiments: H.I. Performed the experiments: H.I. Analyzed the data: H.I. and N.N. Contributed reagents/materials/analysis tools: H.I., N.N., M.O., and M.M. All authors have read and agreed to the published version of the manuscript.

Funding: This study was funded by KAKENHI (16H06892 and 19K15861 to HI, 20H00420 to MI-M).

Acknowledgments: We thank the Tsushima Wildlife Conservation Center and the Japanese Ministry of the Environment for providing samples.

Conflicts of Interest: The authors declare no conflict of interest. The funders had no role in the design of the study; in the collection, analyses, or interpretation of data; in the writing of the manuscript, or in the decision to publish the results.

\section{References}

1. Ross, J.; Brodie, J.; Cheyne, S.; Hearn, A.; Izawa, M.; Loken, B.; Lynam, A.; McCarthy, J.; Mukherjee, S.; Phan, C.; et al. Prionailurus bengalensis. Available online: https://www.iucnredlist.org/species/18146/50661611 (accessed on 20 July 2020).

2. Wozencraft, W. Order Carnivora. In Mammal Species of the World: A Taxonomic and Geographic Reference, 3rd ed.; Wilson, D., Reeder, D., Eds.; Johns Hopkins University Press: Baltimore, MD, USA, 2005; pp. 532-628.

3. Patel, R.P.; Wutke, S.; Lenz, D.; Mukherjee, S.; Ramakrishnan, U.; Veron, G.; Fickel, J.; Wilting, A.; Forster, D.W. Genetic Structure and Phylogeography of the Leopard Cat (Prionailurus bengalensis) Inferred from Mitochondrial Genomes. J. Hered. 2017, 108, 349-360. [CrossRef]

4. Izawa, M.; Doi, T.; Nakanishi, N.; Teranishi, A. Ecology and conservation of two endangered subspecies of the leopard cat (Prionailurus bengalensis) on Japanese islands. Biol. Conserv. 2009, 142, 1884-1890. [CrossRef]

5. Masuda, R.; Yoshida, M.C. Two Japanese wildcats, the Tsushima cat and the Iriomote cat, show the same mitochondrial DNA lineage as the leopard cat Felis bengalensis. Zool. Sci. 1995, 12, 655-659. [CrossRef] [PubMed]

6. Tamada, T.; Siriaroonrat, B.; Subramaniam, V.; Hamachi, M.; Lin, L.K.; Oshida, T.; Rerkamnuaychoke, W.; Masuda, R. Molecular diversity and phylogeography of the Asian leopard cat, Felis bengalensis, inferred from mitochondrial and Y-chromosomal DNA sequences. Zool. Sci. 2008, 25, 154-163. [CrossRef] [PubMed]

7. Ito, H.; Inoue-Murayama, M. The Tsushima leopard cat exhibits extremely low genetic diversity compared with the Korean Amur leopard cat: Implications for conservation. Peer] 2019, 7, e7297. [CrossRef]

8. Russello, M.A.; Amato, G. Ex situ population management in the absence of pedigree information. Mol. Ecol. 2004, 13, 2829-2840. [CrossRef]

9. Ellegren, H. Microsatellites: Simple sequences with complex evolution. Nat. Rev. Genet. 2004, 5, 435-445. [CrossRef]

10. Schlotterer, C. Evolutionary dynamics of microsatellite DNA. Chromosoma 2000, 109, 365-371. [CrossRef]

11. Guichoux, E.; Lagache, L.; Wagner, S.; Chaumeil, P.; Léger, P.; Lepais, O.; Lepoittevin, C.; Malausa, T.; Revardel, E.; Salin, F.; et al. Current trends in microsatellite genotyping. Mol. Ecol. Resour. 2011, 11, 591-611. [CrossRef]

12. Garvin, M.R.; Saitoh, K.; Gharrett, A.J. Application of single nucleotide polymorphisms to non-model species: A technical review. Mol. Ecol. Resour. 2010, 10, 915-934. [CrossRef] [PubMed]

13. Helyar, S.J.; Hemmer-Hansen, J.; Bekkevold, D.; Taylor, M.I.; Ogden, R.; Limborg, M.T.; Cariani, A.; Maes, G.E.; Diopere, E.; Carvalho, G.R.; et al. Application of SNPs for population genetics of nonmodel organisms: New opportunities and challenges. Mol. Ecol. Resour. 2011, 11 (Suppl. 1), 123-136. [CrossRef] [PubMed]

14. Baird, N.A.; Etter, P.D.; Atwood, T.S.; Currey, M.C.; Shiver, A.L.; Lewis, Z.A.; Selker, E.U.; Cresko, W.A.; Johnson, E.A. Rapid SNP Discovery and Genetic Mapping Using Sequenced RAD Markers. PLoS ONE 2008, 3, e3376. [CrossRef] [PubMed]

15. Lavretsky, P.; Janzen, T.; McCracken, K.G. Identifying hybrids \& the genomics of hybridization: Mallards \& American black ducks of Eastern North America. Ecol. Evol. 2019, 9, 3470-3490. [CrossRef] [PubMed] 
16. Suyama, Y.; Matsuki, Y. MIG-seq: An effective PCR-based method for genome-wide single-nucleotide polymorphism genotyping using the next-generation sequencing platform. Sci. Rep. 2015, 5, 16963. [CrossRef]

17. Campbell, N.R.; Harmon, S.A.; Narum, S.R. Genotyping-in-Thousands by sequencing (GT-seq): A cost effective SNP genotyping method based on custom amplicon sequencing. Mol. Ecol. Resour. 2015, 15, 855-867. [CrossRef]

18. Schmidt, D.; Campbell, N.R.; Govindarajulu, P.; Larsen, K.W.; Russello, M.A. Genotyping-in-Thousands by sequencing (GT-seq) panel development and application to minimally-invasive DNA samples to support studies in molecular ecology. Mol. Ecol. Resour. 2019. [CrossRef]

19. Hosoya, S.; Hirase, S.; Kikuchi, K.; Nanjo, K.; Nakamura, Y.; Kohno, H.; Sano, M. Random PCR-based genotyping by sequencing technology GRAS-Di (genotyping by random amplicon sequencing, direct) reveals genetic structure of mangrove fishes. Mol. Ecol. Resour. 2019, 19, 1153-1163. [CrossRef]

20. Enoki, H.; Takeuchi, Y. New Genotyping Technology, GRAS-Di, Using Next Generation Sequencer. In Proceedings of the Plant and Animal Genome XXVI Conference, San Diego, CA, USA, 13-17 January 2018.

21. Roques, S.; Chancerel, E.; Boury, C.; Pierre, M.; Acolas, M.L. From microsatellites to single nucleotide polymorphisms for the genetic monitoring of a critically endangered sturgeon. Ecol. Evol. 2019, 9, 7017-7029. [CrossRef]

22. Enoki, H. The construction of psedomolecules of a commercial strawberry by DeNovoMAGIC and new genotyping technology, GRAS-Di. In Proceedings of the Plant and Animal Genome Conference XXVII, San Diego, CA, USA, 12-16 January 2019.

23. Joshi, N.A.; Fass, J.N. Sickle: A Sliding-Window, Adaptive, Quality-Based Trimming Tool for FastQ Files (Version 1.33) [Software]. Available online: https://github.com/najoshi/sickle (accessed on 1 December 2019).

24. Gordon, A.; Hannon, G.J. Fastx-Toolkit. FASTQ/A Short-Reads Preprocessing Tools. Available online: http://hannonlab.cshl.edu/fastx_toolkit (accessed on 1 December 2019).

25. Rochette, N.C.; Rivera-Colon, A.G.; Catchen, J.M. Stacks 2: Analytical methods for paired-end sequencing improve RADseq-based population genomics. Mol. Ecol. 2019. [CrossRef]

26. Ikeda, H.; Yakubov, V.; Barkalov, V.; Sato, K.; Fujii, N. East Asian origin of the widespread alpine snow-bed herb, Primula cuneifolia (Primulaceae), in the northern Pacific region. J. Biogeogr. 2020. [CrossRef]

27. Li, H.; Durbin, R. Fast and accurate short read alignment with Burrows-Wheeler transform. Bioinformatics 2009, 25, 1754-1760. [CrossRef] [PubMed]

28. Li, H.; Handsaker, B.; Wysoker, A.; Fennell, T.; Ruan, J.; Homer, N.; Marth, G.; Abecasis, G.; Durbin, R.; Genome Project Data Processing Subgroup. The Sequence Alignment/Map format and SAMtools. Bioinformatics 2009, 25, 2078-2079. [CrossRef] [PubMed]

29. Li, H. Improving SNP discovery by base alignment quality. Bioinformatics 2011, 27, 1157-1158. [CrossRef] [PubMed]

30. Wala, J.; Zhang, C.Z.; Meyerson, M.; Beroukhim, R. VariantBam: Filtering and profiling of next-generational sequencing data using region-specific rules. Bioinformatics 2016, 32, 2029-2031. [CrossRef]

31. Chang, C.C.; Chow, C.C.; Tellier, L.C.; Vattikuti, S.; Purcell, S.M.; Lee, J.J. Second-generation PLINK: Rising to the challenge of larger and richer datasets. Gigascience 2015, 4. [CrossRef] [PubMed]

32. Lischer, H.E.L.; Excoffier, L. PGDSpider: An automated data conversion tool for connecting population genetics and genomics programs. Bioinformatics 2011, 28, 298-299. [CrossRef] [PubMed]

33. Peakall, R.; Smouse, P.E. GenAlEx 6.5: Genetic analysis in Excel. Population genetic software for teaching and research-An update. Bioinformatics 2012, 28, 2537-2539. [CrossRef]

34. Chhatre, V.E.; Emerson, K.J. StrAuto: Automation and parallelization of STRUCTURE analysis. BMC Bioinform. 2017, 18, 192. [CrossRef]

35. Pritchard, J.K.; Stephens, M.; Donnelly, P. Inference of population structure using multilocus genotype data. Genetics 2000, 155, 945-959.

36. Evanno, G.; Regnaut, S.; Goudet, J. Detecting the number of clusters of individuals using the software structure: A simulation study. Mol. Ecol. 2005, 14, 2611-2620. [CrossRef]

37. Earl, D.; von Holdt, B. Structure Harvester: A website and program for visualizing STRUCTURE output and implementing the Evanno method. Conserv. Genet. Resour. 2012, 4, 359-361. [CrossRef]

38. Wang, J. A parsimony estimator of the number of populations from a STRUCTURE-like analysis. Mol. Ecol. Resour. 2019, 19, 970-981. [CrossRef] [PubMed] 
39. Kopelman, N.M.; Mayzel, J.; Jakobsson, M.; Rosenberg, N.A.; Mayrose, I. Clumpak: A program for identifying clustering modes and packaging population structure inferences across K. Mol. Ecol. Resour. 2015, 15, 1179-1191. [CrossRef] [PubMed]

40. Tateno, M.; Nishio, T.; Matsuo, T.; Sakuma, M.; Nakanishi, N.; Izawa, M.; Asari, Y.; Okamura, M.; Miyama, T.S.; Setoguchi, A.; et al. Epidemiological Survey of Tick-Borne Protozoal Infection in Iriomote Cats and Tsushima Leopard Cats in Japan. J. Vet. Med. Sci. 2013. [CrossRef] [PubMed]

41. Saka, T.; Nishita, Y.; Masuda, R. Low genetic variation in the MHC class II DRB gene and MHC-linked microsatellites in endangered island populations of the leopard cat (Prionailurus bengalensis) in Japan. Immunogenetics 2017. [CrossRef] [PubMed]

42. Adachi, I.; Kusuda, S.; Nagao, E.; Taira, Y.; Asano, M.; Tsubota, T.; Doi, O. Fecal steroid metabolites and reproductive monitoring in a female Tsushima leopard cat (Prionailurus bengalensis euptilurus). Theriogenology 2010, 74, 1499-1503. [CrossRef]

43. Pecoraro, C.; Babbucci, M.; Villamor, A.; Franch, R.; Papetti, C.; Leroy, B.; Ortega-Garcia, S.; Muir, J.; Rooker, J.; Arocha, F.; et al. Methodological assessment of 2b-RAD genotyping technique for population structure inferences in yellowfin tuna (Thunnus albacares). Mar. Genomics 2016, 25, 43-48. [CrossRef]

44. DiBattista, J.D.; Saenz-Agudelo, P.; Piatek, M.J.; Wang, X.; Aranda, M.; Berumen, M.L. Using a butterflyfish genome as a general tool for RAD-Seq studies in specialized reef fish. Mol. Ecol. Resour. 2017, 17, 1330-1341. [CrossRef]

45. Shafer, A.B.A.; Peart, C.R.; Tusso, S.; Maayan, I.; Brelsford, A.; Wheat, C.W.; Wolf, J.B.W. Bioinformatic processing of RAD-seq data dramatically impacts downstream population genetic inference. Methods Ecol. Evol. 2017, 8, 907-917. [CrossRef]

46. Rochette, N.C.; Catchen, J.M. Deriving genotypes from RAD-seq short-read data using Stacks. Nat. Protoc. 2017, 12, 2640-2659. [CrossRef]

47. Díaz-Arce, N.; Rodríguez-Ezpeleta, N. Selecting RAD-Seq Data Analysis Parameters for Population Genetics: The More the Better? Front. Genet. 2019, 10, 533. [CrossRef] [PubMed]

48. Paris, J.R.; Stevens, J.R.; Catchen, J.M. Lost in parameter space: A road map for stacks. Methods Ecol. Evol. 2017, 8, 1360-1373. [CrossRef]

49. Zhang, W.Q.; Zhang, M.H. Complete mitochondrial genomes reveal phylogeny relationship and evolutionary history of the family Felidae. Genet. Mol. Res. 2013, 12, 3256-3262. [CrossRef] [PubMed]

50. Gillespie, J.H. Population Genetics: A Concise Guide; Johns Hopkins University Press: Baltimore, MD, USA, 2004.

51. Waples, R.S. Testing for Hardy-Weinberg proportions: Have we lost the plot? J. Hered. 2015, 106, 1-19. [CrossRef]

52. Wigginton, J.E.; Cutler, D.J.; Abecasis, G.R. A note on exact tests of Hardy-Weinberg equilibrium. Am. J. Hum. Genet. 2005, 76, 887-893. [CrossRef]

53. Yu, C.; Zhang, S.; Zhou, C.; Sile, S. A likelihood ratio test of population Hardy-Weinberg equilibrium for case-control studies. Genet. Epidemiol. 2009, 33, 275-280. [CrossRef]

54. Anderson, C.A.; Pettersson, F.H.; Clarke, G.M.; Cardon, L.R.; Morris, A.P.; Zondervan, K.T. Data quality control in genetic case-control association studies. Nat. Protoc. 2010, 5, 1564-1573. [CrossRef]

55. Graffelman, J.; Jain, D.; Weir, B. A genome-wide study of Hardy-Weinberg equilibrium with next generation sequence data. Hum. Genet. 2017, 136, 727-741. [CrossRef]

56. Janes, J.K.; Miller, J.M.; Dupuis, J.R.; Malenfant, R.M.; Gorrell, J.C.; Cullingham, C.I.; Andrew, R.L. The K= 2 conundrum. Mol. Ecol. 2017, 26, 3594-3602. [CrossRef]

57. Ito, H.; Ogden, R.; Langenhorst, T.; Inoue-Murayama, M. Contrasting results from molecular and pedigree-based population diversity measures in captive zebra highlight challenges facing genetic management of zoo populations. Zoo Biol. 2017, 36, 87-94. [CrossRef]

(C) 2020 by the authors. Licensee MDPI, Basel, Switzerland. This article is an open access article distributed under the terms and conditions of the Creative Commons Attribution (CC BY) license (http://creativecommons.org/licenses/by/4.0/). 